DRAFT MANUSCRIPT PREPARED FOR SUBMISSION TO THE 21ST INTERNATIONAL CONGRESS ON ACOUSTICS, MONTREAL, 2-7 JUNE 2013

\title{
The influence of regularization on anechoic performance and robustness of sound zone methods
}

\author{
Philip Coleman, Philip Jackson, Marek Olik \\ Centre for Vision, Speech and Signal Processing, University of Surrey, Guildford, Surrey, GU2 \\ $7 X H$ \\ Martin Olsen, Martin Møller, Jan Abildgaard Pedersen \\ Bang \& Olufsen A / S, Peter Bangs Vej 15, DK7600, Struer, Denmark
}

February 4, 2013

\begin{abstract}
Recent attention to the problem of controlling multiple loudspeakers to create sound zones has been directed towards practical issues arising from system robustness concerns. In this study, the effects of regularization are analyzed for three representative sound zoning methods. Regularization governs the control effort required to drive the loudspeaker array, via a constraint in each optimization cost function. Simulations show that regularization has a significant effect on the sound zone performance, both under ideal anechoic conditions and when systematic errors are introduced between calculation of the source weights and their application to the system. Results are obtained for speed of sound variations and loudspeaker positioning errors with respect to the source weights calculated. Judicious selection of the regularization parameter is shown to be a primary concern for sound zone system designers - the acoustic contrast can be increased by up to $50 \mathrm{~dB}$ with proper regularization in the presence of errors. A frequency-dependent minimum regularization parameter is determined based on the conditioning of the matrix inverse. The regularization parameter can be further increased to improve performance depending on the effort constraints, expected magnitude of errors, and desired sound field properties of the system.
\end{abstract}




\section{INTRODUCTION}

Array signal processing techniques for sound zoning are derived from two approaches: sound field synthesis (which can be solved analytically [e.g. 1] or by directly optimizing the complex pressures in the zones [e.g. 2]), where the entire sound field controlled by the array can be specified, and beamforming [e.g. 3]. The latter approach extends to energy cancellation methods which have been used extensively for the sound zone problem [e.g. 3; 4; 5]. Hybrids between the above techniques have also been proposed [e.g. $6 ; 7]$.

The sound field synthesis techniques are characterized by accurate reproduction of the target sound field, although this can be at the cost of excessive control effort [2] and zone contrast performance [1]. Conversely, the energy cancellation techniques are phase-ambiguous and the wave propagation in the sound field is uncontrolled [1], but they produce excellent cancellation. Beamforming (with no cancellation) has a low effort cost but does not produce significant contrast.

Many methods for determining the value of a frequency-dependent Tikhonov regularization parameter have been proposed, based on a hard effort constraint [8], fixed eigenvalue ratio [9] or optimal trade-offs between effort and reproduction error (e.g. the L-curve and Generalised Cross-Validation [10]). The effect of the Tikhonov regularization is comparable with using a pseudo-inverse approach (based on a truncated singular value decomposition) and modifying the threshold for a singular value being discarded, but the modal control is more continuous using the regularization approach and it has a clearer physical definition.

With such techniques available, the challenge remains to implement a system that is as robust as possible. Sound zone implementations require robustness to many kinds of degradations, for example scattering, measurement noise and varying experimental conditions. The robustness of some techniques to errors has been considered in the literature for scattering [11] and errors in the setup [12] and playback [8] transfer functions. However, system robustness, regularization and the corresponding effort have not been compared amongst approaches under uniform conditions. Here, we introduce the cost functions for three representative methods: brightness control (beamforming), acoustic contrast control (energy cancellation) and pressure matching (sound field synthesis). The effect of the regularization parameter on effort and robustness to systematic errors is explored through anechoic computer simulations focusing on the effect of varying experimental conditions, and a method for establishing the regularization parameter is proposed.

\section{SOUND ZONE EVALUATION}

The acoustic contrast between target zone A and dark zone B is defined as the ratio of sound pressures in each zone due to the reproduction of program A, expressed in decibels (for a single frequency):

$$
\text { contrast }_{A B}=\bar{p}_{S P L_{A}}-\bar{p}_{S P L_{B}},
$$

where $\bar{p}_{S P L_{A}}$ and $\bar{p}_{S P L_{B}}$ denote the spatially averaged sound pressure levels in zones $\mathrm{A}$ and $\mathrm{B}$, respectively, with reference to the threshold of hearing. The contrast achieved between the zones is the primary indication of how effective a sound zone system will be at isolating an audio program.

The control effort is the energy that the loudspeaker array requires to achieve the reproduced sound field. It is defined as the total array energy in terms of the source weight vector $\boldsymbol{q}$, relative to a single reference monopole $q_{r}$ producing the same pressure in the target zone [4], and is expressed in decibels as

$$
\text { effort }_{A}=10 \log _{10}\left(\frac{\boldsymbol{q}^{H} \boldsymbol{q}}{q_{r}^{H} q_{r}}\right) .
$$


The planarity of the sound field - the extent to which the sound field in the target zone resembles a plane wave at a single frequency, is a physical measure recently proposed by Jackson and Jacobsen [13]. The energy distribution at the microphone array over incoming plane wave direction is given by $w_{i}=\frac{1}{2} \psi_{i}^{*} \psi_{i}$, where.$^{*}$ denotes the complex conjugate, $\boldsymbol{w}=\left[w_{1} \ldots w_{i}\right]$ are the energy components and $\boldsymbol{\psi}=\boldsymbol{H p}$ is the distribution of plane waves over incoming direction, where $\boldsymbol{H}$ is a steering matrix based on the microphone grid. $\boldsymbol{H}$ was populated by a super-directive beamformer, as in [13]. The planarity metric is defined for the target zone as the ratio between the energy due to the largest plane wave component and the total energy flux of plane wave components:

$$
\text { planarity }_{A}=\frac{\sum_{i} w_{i} \boldsymbol{u}_{i} \cdot \boldsymbol{u}_{\hat{\imath}}}{\sum_{i} w_{i}}
$$

where $\boldsymbol{u}_{i}$ is the unit vector associated with the principal component's direction, $\boldsymbol{u}_{\hat{\imath}}$ is the sum of all components in the $\hat{\imath}$ th direction $\hat{\imath}=\arg \max _{i} w_{i}$, and . denotes the inner product.

\section{THEORY}

Brightness control, acoustic contrast control and pressure matching are considered in this study. Brightness control (BC) represents the optimal beamforming approach to producing sound zones. The cost function is written as a constrained optimization problem, maximizing the pressure in the bright zone whilst constraining the effort [3]:

$$
J=\left(\boldsymbol{p}_{b}^{H} \boldsymbol{p}_{b}-B\right)-\lambda\left(\boldsymbol{q}^{H} \boldsymbol{q}-E\right),
$$

where $B$ is the required sound pressure in the bright zone and $E$ is the maximum allowed control effort. The solution can be found by taking the gradient of Eq. (4) and rearranging as an eigenvalue problem, $\lambda \boldsymbol{q}=\left(\boldsymbol{G}_{b}^{H} \boldsymbol{G}_{b}\right) \boldsymbol{q}$, and the optimal $\boldsymbol{q}$ found by taking the eigenvector corresponding to the maximum eigenvalue of $\boldsymbol{G}_{b}^{H} \boldsymbol{G}_{b}$. The Lagrangian multiplier $\lambda$ upon which the solution is found must be directly scaled to achieve either the desired target pressure $B$ or control effort $E$. Here, we fix the target reproduction level $B$ for all simulations, so there is a corresponding fixed value of $\lambda$ and the control effort may differ from $E$.

Acoustic contrast control (ACC) [3] represents the energy cancellation case. Introducing the indirect' Tikhonov regularization proposed by Elliot et al. [8], the cost function is written as a constrained optimization problem, minimizing the pressure in the dark zone whilst constraining the pressure in the bright zone to be as large as possible, with an added effort term to regularize the solution:

$$
J=\boldsymbol{p}_{d}^{H} \boldsymbol{p}_{d}+\lambda_{1}\left(\boldsymbol{p}_{b}^{H} \boldsymbol{p}_{b}-B\right)+\lambda_{2}\left(\boldsymbol{q}^{H} \boldsymbol{q}-E\right),
$$

where $\boldsymbol{p}_{d}=\boldsymbol{G}_{d} \boldsymbol{q}$ is the vector of complex pressures at the microphones in the dark zone, and $\boldsymbol{G}_{d}$ is the plant matrix with respect to the dark zone.

Minimizing the problem as above, the eigenvalue problem becomes $\lambda_{1} \boldsymbol{q}=-\left(\boldsymbol{G}_{b}^{H} \boldsymbol{G}_{b}\right)^{-1}\left(\boldsymbol{G}_{d}^{H} \boldsymbol{G}_{d}+\right.$ $\left.\lambda_{2} \boldsymbol{I}\right) \boldsymbol{q}$. The minimum can be found by taking the eigenvector corresponding to the maximum eigenvalue of $\left(\boldsymbol{G}_{d}^{H} \boldsymbol{G}_{d}+\lambda_{2} \boldsymbol{I}\right)^{-1}\left(\boldsymbol{G}_{b}^{H} \boldsymbol{G}_{b}\right)$ [8]. The regularization term $\lambda_{2}$ therefore regularizes both the effort and the inversion of $\boldsymbol{G}_{b}^{H} \boldsymbol{G}_{b}$.

Pressure matching (PM) represents the sound field synthesis approach. Contrast between the zones can be achieved by setting the pressure amplitudes in one zone to be highly attenuated. The optimization cost function is written to directly minimize the error $\boldsymbol{e}$ between the desired sound field (in this case a plane wave in the target zone and zero pressure amplitude in the dark zone) and reproduced sound field, with an effort constraint for Tikhonov regularization:

$$
J=\boldsymbol{e}^{H} \boldsymbol{e}+\lambda\left(\boldsymbol{q}^{H} \boldsymbol{q}-E\right) .
$$


The optimal solution is $\boldsymbol{q}=\left(\boldsymbol{G}^{H} \boldsymbol{G}+\lambda \boldsymbol{I}\right)^{-1} \boldsymbol{G}^{H} \boldsymbol{d}$, where $\boldsymbol{G}=\left[\boldsymbol{G}_{b} \boldsymbol{G}_{d}\right]^{T}$ is the total system plant matrix. It is noteworthy that by application of the effort constraint $\left(\boldsymbol{q}^{H} \boldsymbol{q}-E\right)$ to the ACC and PM cost functions, the value of $\lambda$ and corresponding effort can be directly compared with that of $\lambda_{2}$ in Eq. (5), even though the solutions are found using different techniques.

\section{Simulations}

The simulations were conducted in Matlab, simulating a free-field lossless anechoic environment, with each source modeled as an ideal monopole. The free-field Green's Function, $g=\frac{j \rho c k q}{4 \pi r} e^{j k r}$, was used to populate the plant matrices. A circular loudspeaker array of radius $1.2 \mathrm{~m}$ comprising 48 equally spaced elements was used for reproduction. The microphones used for calculating the sound zone filters (setup) and those for obtaining predictions (playback) were kept spatially distinct [14] in order to assess the general sound field rather than the performance at the specific points sampled for setup. The microphone geometry was fixed, meaning that the independence of the setup and playback points increased with frequency. A total of 156 omnidirectional microphones with a spacing of $2.1 \mathrm{~cm}$ were arranged in each zone to sample a circle of $30 \mathrm{~cm}$ diameter. The loudspeaker weights were scaled to give the target spatially averaged SPL of $76 \mathrm{~dB}$, which has been used during listening tests based on the sound zone interference situation [15]. Sound pressures below $0 \mathrm{~dB}$ are not considered (there is also no noise floor) and therefore an upper contrast limit of $76 \mathrm{~dB}$ is imposed.

\section{Varying the regularization conditions}

Regularization has two key functions: to improve the condition number of the matrix for inversion (reducing the impact of numerical errors), and to constrain the effort required by the array to reproduce the specified sound field (reducing the overall sound energy in the enclosure and thereby the impact of reflections in a real room, limiting the drive of each loudspeaker resulting in more realizable filters, and reducing the influence of calibration/setup errors). If the regularization parameter is too small, the conditioning of the matrix will remain poor and the effort may be excessive. If it is too large, the effort will be well controlled but significant errors in the solution will reduce the contrast performance. Furthermore, the condition number of the matrix is highly dependent on the system geometry and varies as a function of frequency.

First, the regularization was tested under ideal conditions with assumed perfect estimates of the system's acoustic response. The regularization parameter was varied from $10^{-10}$ to $10^{10}$ at 1000 logarithmically spaced values. Figure 1 shows the effect of regularization on the contrast, effort and planarity reproduced by the array. Whilst the parameter cannot be varied for BC, the scores are plotted at the point where they intersect with the value of $\lambda$ (Eq. (4)) required to achieve the target sound pressure in the bright zone.

There are three regions of performance in relation to the effort. First, for very small regularization parameters, numerical errors in the matrix inversion cause an unstable effort response, most clearly visible at $500 \mathrm{~Hz}$, and can also be observed in e.g. ACC planarity and PM contrast. In the second region, there is a monotonic relationship between increasing the regularization parameter and decreasing effort. Finally, the minimum possible effort is reached. The asymptotic minimum effort values correspond to the BC effort values, showing this to be least-effort approach, albeit with poor contrast. The amount of regularization necessary to constrain the effort to a particular value can also be observed. It is clear that in order to constrain PM to the same range of effort scores as ACC, the regularization parameter must be very large.

Whilst an increased regularization parameter consistently reduces the effort for each method, the 

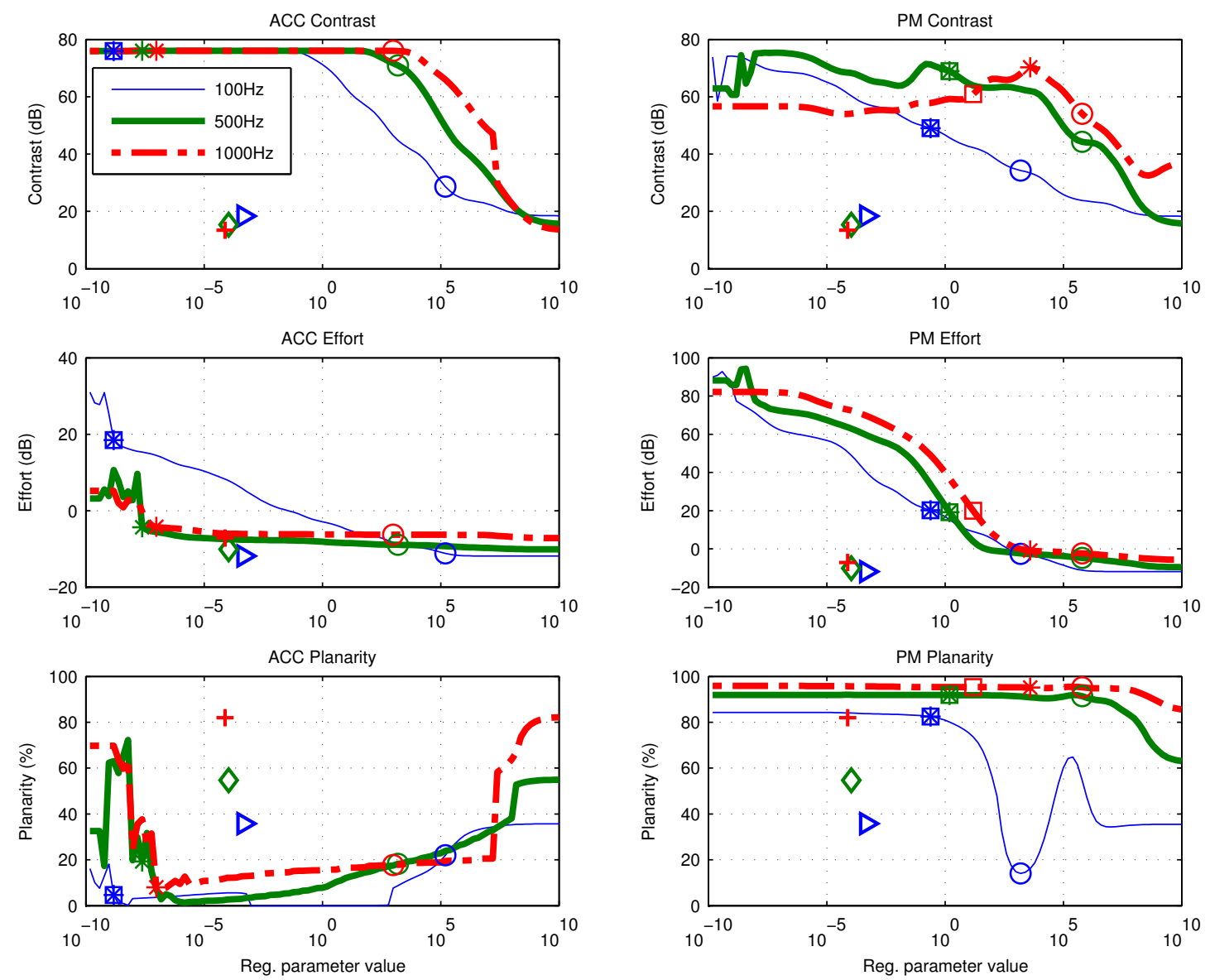

FiguRE 1: (Color online) Performance of acoustic contrast control (left column) and pressure matching (right column) as function of the regularization parameter, in terms of the contrast achieved (top row), effort (middle row) and planarity (bottom row), at $100 \mathrm{~Hz}$ (thin), $500 \mathrm{~Hz}$ (thick), and $1 \mathrm{kHz}$ (thick, dot-dash). The brightness control scores are indicated for each frequency $(100 \mathrm{~Hz} \triangleright ; 500 \mathrm{~Hz} \diamond ; 1000 \mathrm{~Hz}+)$ as single points where they intersect with the $\lambda$ value required to reproduce the target SPL in the bright zone. The regularization parameters corresponding to the effort limit $(\square)$, L-curve (०) and proposed (*) approaches are marked on each line.

relationship with contrast varies. BC gives the lower performance bound and PM and ACC both tend towards this score for very high regularization parameter values. For ACC, the regularization has no noticeable effect on the upper performance, until the regularization error eventually causes the contrast to degrade. For PM, there are local maxima in the contrast, becoming increasingly significant with increasing frequency. In practice, these peaks correspond to the situation where the source weights are physically constrained towards operating as a directive beamformer towards the bright zone.

The choice of regularization parameter has little bearing on the planarity scores once the matrix inversion has been stabilized. The maximum achievable planarity score is constrained by the aperture of the monitor microphone array with relation to the wavelength [13], which accounts for the increase in the maximum PM scores with frequency (at the maximum planarity, a plane wave is accurately reproduced). For very large regularization parameters ACC planarity increases towards the BC score as the array effort is heavily constrained, otherwise the array is typically self-cancelling and the planarity is very poor. PM planarity begins to decrease as the regularization reduces the number of available array modes below that required for accurate reproduction (notable at $100 \mathrm{~Hz}$ ), with the tolerance therefore increasing with frequency.

In Fig. 1, the regularization parameters calculated by three different approaches are marked for reference. These are: Effort limit $(\square)$; adjusting the regularization parameter to enforce a maxi- 
mum allowed array effort (here, as in [8], and by way of an example of this kind of approach, we use a limit of $20 \mathrm{~dB}$ ). L-curve (o); finding the corner of the L-curve based on the reproduction error in terms of the mean-squared difference between the reproduced sound pressure magnitude and the target pressure magnitude. This was used rather than the traditional complex reproduction error so that it could be reasonably applied to ACC which does not control the phase of the sound field. Proposed regularization (*); first applying sufficient regularization to reduce the matrix condition number to the desired level (in this case $10^{16}$ to avoid numerical rounding errors in Matlab), then further increasing the regularization to impose an effort limit, and finally searching along the predicted contrast scores to test whether further increases improve the contrast.

The proposed regularization method can be noted from Fig. 1 to have advantages over the other two methods. The effort limit approach is physically informed, but applying the limit strictly can result in situations where no regularization is applied at all (e.g. ACC $500 \mathrm{~Hz}$ ). Yet, regularization is required to stabilize the performance. Similarly, this technique may under-regularize if there is any performance benefit to further increasing the regularization (e.g. PM $1000 \mathrm{~Hz}$ ). Conversely, the L-Curve tends to over-regularize in relation to the contrast (e.g. PM $1000 \mathrm{~Hz}$ ) and planarity reproduction (e.g. PM $100 \mathrm{~Hz}$ ) due to the target zone errors dominating the curve and a great restriction on effort required. This method tends not to consider absolute effort requirements and may sacrifice performance (which could be improved with a still tolerable effort) or indeed result in effort values above the threshold. In contrast, the proposed method is both physically informed by the numerical and geometrical problem conditioning and the effort threshold, and also incorporates a search based on further regularization increases. For ACC, this ensures that a minimum regularization is achieved, and for PM, finds the best contrast performance within the physical system constraints.

\section{Robustness to mismatched setup and playback conditions}

Even under simulated anechoic conditions, one can see the practical benefits of regularization in relation to the robustness of the system by introducing perturbations. A sound zone system should be robust to small changes in the reproduction atmosphere, and allow some tolerance to the positioning of the equipment, which in practical scenarios will generally be restricted to loudspeaker placement. Therefore, systematic variations to the sound propagation speed and random errors to each loudspeaker position were applied and the performance of the control methods evaluated with various regularization parameters. As the key metric across sound zone systems (assessing the fundamental ability to create sound separation), only the contrast is considered here. After calculating the source weights for a particular array and environment, the configuration was modified before application of the original source weights, thus introducing an error between setup and playback.

First, robustness to sound propagation speed was investigated. This varies with temperature, air pressure and humidity in practical situations. The transfer functions were modified on playback by introducing a variation of up to $10 \mathrm{~m} / \mathrm{s}$ (corresponding to a change in temperature of $17^{\circ} \mathrm{C}$ ) to the Green's function and recalculating the transfer function matrix accordingly. Such a variation, applied consistently across each transfer function term, is analogous to a shift in frequency between setup and playback.

Figure 2a shows the acoustic contrast achieved under the mismatched propagation speed conditions at $100 \mathrm{~Hz}$ and $1000 \mathrm{~Hz}$. It is clear that such error has the potential to seriously degrade the realizable contrast of a system. The various regularization parameters can be seen to have a similar effect between the two methods of ACC and PM at low frequencies, but remarkably different outcomes at higher frequencies. For the mismatched speed of sound at $100 \mathrm{~Hz}$ a very small amount of regularization improves the performance of ACC (but the score is more sensitive to 

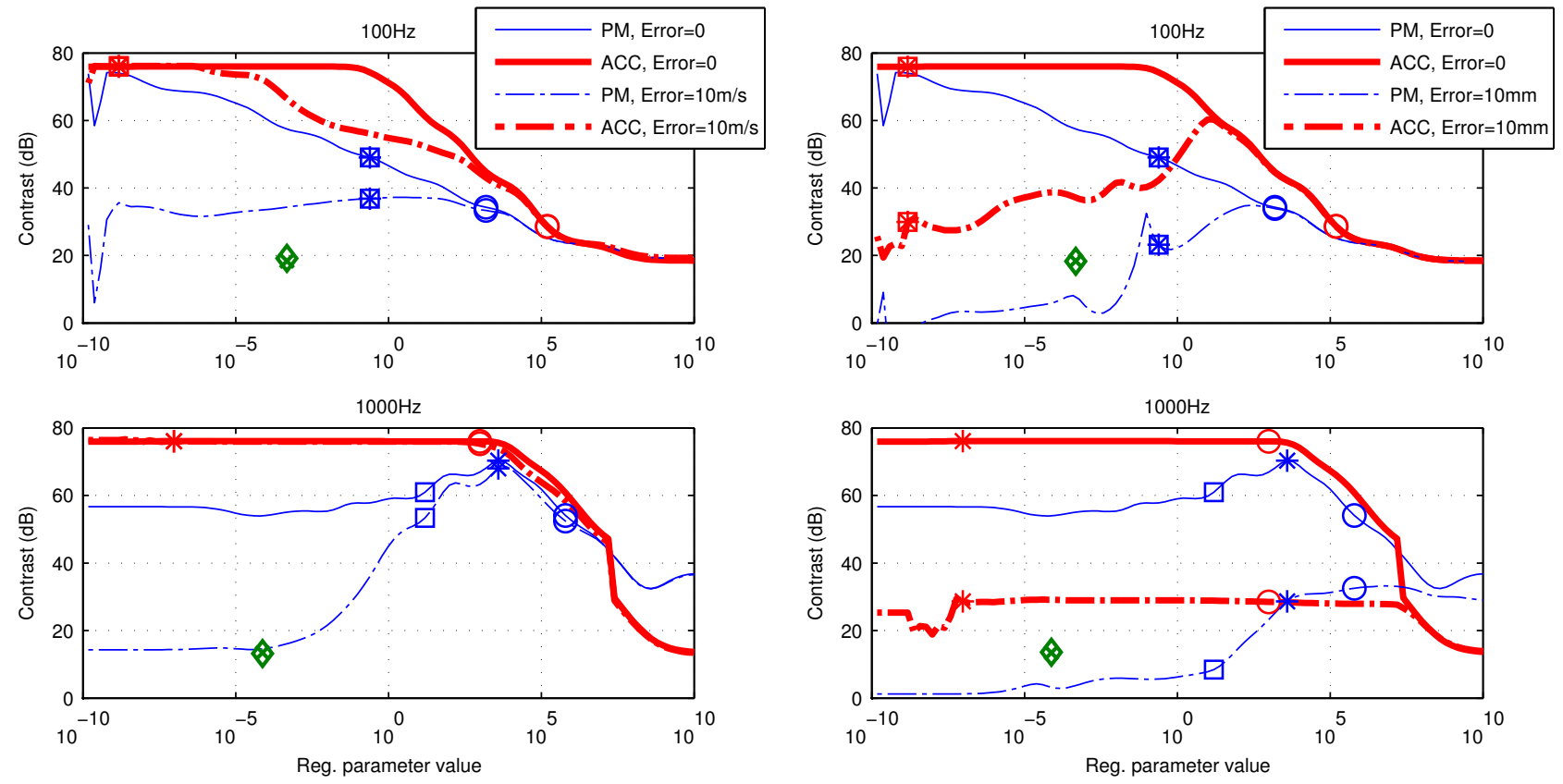

(A) Sound propagation speed error

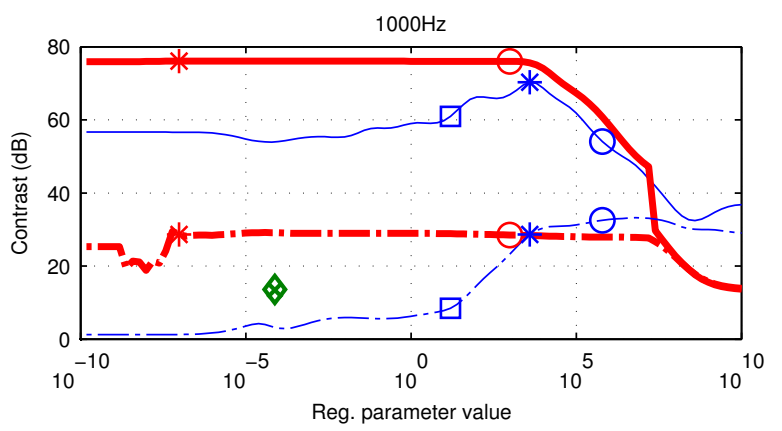

(B) Loudspeaker positioning error

FIGURE 2: (Color online) Effect of the regularization parameter on acoustic contrast at $100 \mathrm{~Hz}$ (top) and $1000 \mathrm{~Hz}$ (bottom), with mismatched playback conditions. The ideal cases for PM (thin) and ACC (thick) are compared with the error cases (thin, dot-dash; and thick, dot-dash; respectively). The brightness control scores with $(\diamond)$ and without $(x)$ the same magnitude of error are also shown as single points where they intersect with the $\lambda$ value required to reproduce the target SPL in the bright zone. The regularization parameters corresponding to the effort limit ( $\square$ ), L-curve (o) and proposed (*) approaches are marked on each line.

increased regularization overall), and the PM performance is slightly improved by increasing the regularization. At $1000 \mathrm{~Hz}$, the effect of the error on ACC is negligible for all regularization parameters. For PM, on the other hand, regularization has a significant effect on the contrast, and the performance degradation of over $40 \mathrm{~dB}$ from the ideal case can be almost entirely removed, with optimal regularization giving over $50 \mathrm{~dB}$ performance improvement from the unregularized case. The best robustness to error is given at the point of optimal regularization calculated for the ideal case using the proposed method.

The second mismatch introduced between the setup and playback of the source weights was a variation in the positioning of the loudspeakers. Each loudspeaker was moved independently in the $x$ and $y$ directions by a random amount drawn from a normal distribution. Unlike the systematic error in sound propagation speed, the error on the phase component of the transfer function is not the same for each path, and additionally an amplitude error is introduced. Here, the maximum error considered was with one standard deviation of the loudspeaker placement equal to $10 \mathrm{~mm}$. The $95 \%$ confidence interval has a diameter in the $\mathrm{x}-\mathrm{y}$ plane of $57 \mathrm{~mm}$ about the setup location, which might correspond to re-installation of a large sound zone system without precise positioning instruments. For a rigidly installed system (e.g. a sound system in a car), considerably smaller variation in loudspeaker locations would be expected.

Figure $2 \mathrm{~b}$ shows the results in comparison with the ideal case. As with the case of the propagation speed error, the behavior between the methods at $100 \mathrm{~Hz}$ is fairly comparable, yet at $1000 \mathrm{~Hz}$ it varies considerably. At $100 \mathrm{~Hz}$, the degradation is seen to be generally more severe for PM than it is for ACC, as for the propagation speed results at this frequency. In this case, however, the performance error for PM is so severe that the contrast becomes negative for very light regularization. Increasing the regularization of ACC brings about a significant improvement of $40 \mathrm{~dB}$ 


\begin{tabular}{|l|l|l|l|l|l|l|}
\hline \multirow{2}{*}{ Conditions } & \multicolumn{2}{|c|}{ 20dB Limit } & \multicolumn{2}{c|}{ L-Curve } & \multicolumn{2}{c|}{ Proposed } \\
\cline { 2 - 8 } & ACC & PM & ACC & PM & ACC & PM \\
\hline Ideal & $\mathbf{7 6}$ & 61 & $\mathbf{7 6}$ & 54 & $\mathbf{7 6}$ & 70 \\
\hline Speed of sound error (10m/s) & $\mathbf{7 6}$ & 53 & 75 & 52 & $\mathbf{7 6}$ & 67 \\
\hline Loudspeaker position error (s.d. 10mm) & 25 & 8 & 29 & $\mathbf{3 3}$ & 28 & 29 \\
\hline
\end{tabular}

TABLE 1: Summary contrast scores (to the nearest $\mathrm{dB}$ ) comparing ACC and PM performance and regularization schemes for the various error conditions, at $1 \mathrm{kHz}$.

in the contrast. PM is unable to control the sound field until a suitable regularization point is reached, and the optimal point coincides with the point where the lines converge. The optimal ACC contrast is $60 \mathrm{~dB}$, while the optimal PM contrast is $35 \mathrm{~dB}$. However, none of the regularization approaches considered under ideal conditions find the optimal contrast point when the system is subject to error, although the L-curve derived parameter gives a reasonable result for PM.

At $1000 \mathrm{~Hz}$, increasing the regularization for ACC above that given by the proposed method does not bring about any further benefit in contrast. However, PM behaves in a similar manner to the lower frequency case, where there is severe degradation for light regularization, and very large regularization parameters can improve performance. At the maximum point, the optimal contrast achieved by regularized PM becomes favorable over ACC by $5 \mathrm{~dB}$. Therefore, for ACC there is little that can be done by regularization to improve the robustness to this kind of error once the matrix inversion has been adequately conditioned, but for PM the effect remains. At $1000 \mathrm{~Hz}$, both the optimal and L-curve methods of calculating the regularization parameter give reasonable results, with the L-curve achieving $5 \mathrm{~dB}$ more contrast. If the system were able to predict the contrast performance subject to this kind of error, for instance by testing on independent measurement sets, it may be possible to implement a search approach similar to that used in the proposed regularization method to improve the ACC low frequency robustness. It is nevertheless interesting to note the susceptibility of ACC to these kinds of errors and, at higher frequencies, its inability to recover from them by employing regularization.

Table 1 shows a summary of the contrast scores for the various regularization. For position error, all contrast scores are severely degraded and there is a maximum $4 \mathrm{~dB}$ difference between the methods for the L-Curve and proposed regularization, with neither finding the exact optimum. However, significant improvements can be obtained under ideal and speed of sound error conditions by using the proposed regularization method, and ACC gives the greater contrast score.

\section{Conclusions}

The simulations presented here highlight the importance of judicious selection of the regularization parameter for optimal sound zone performance, even under ideal anechoic conditions. Under mismatched setup and playback conditions, the contrast is markedly reduced, even for relatively small errors. Regularization can recover good performance when the sound propagation speed is mismatched, but when the loudspeaker positions are varied the regularization can only limit the degradation. For increasing error, the optimal contrast scores between ACC and PM become very close. To determine the optimal regularization, the parameter should be initially set to satisfactorily condition the matrix inversion, further increased to enforce a physical limit on system effort and the contrast gradient checked to determine if any advantage can be gained by further increases in regularization. In some cases, the anechoic contrast can be improved by over $50 \mathrm{~dB}$ by following this approach, compared to the unregularized case, and an improvement in contrast can be made over the L-curve approach which tends to over-regularize. Future work will investigate the influence of regularization on the performance of real-world sound zone implementations. 


\section{ACKNOWLEDGEMENTS}

This research was supported by Bang \& Olufsen A/S and an EPSRC Doctoral Training Grant, and forms part of the POSZ project (www.posz.org).

\section{REFERENCES}

[1] F. Jacobsen, M. Olsen, M. Møller, and F. Agerkvist, "A comparison of two strategies for generating sound zones in a room", in Proceedings of the 18th International Congress on Sound and Vibration (International Institute of Acoustics and Vibration) (2011).

[2] M. Poletti, "An investigation of 2-d multizone surround sound systems", in Proceedings of the 125th Audio Engineering Society Convention (2008).

[3] J. Choi and Y. Kim, "Generation of an acoustically bright zone with an illuminated region using multiple sources”, J. Acoust. Soc. Am. 111, 1695-1700 (2002).

[4] S. J. Elliott, J. Cheer, H. Murfet, and K. R. Holland, "Minimally radiating sources for personal audio”, J. Acoust. Soc. Am. 128, 1721-1728 (2010).

[5] M. Shin, S. Q. Lee, F. M. Fazi, P. A. Nelson, D. Kim, S. Wang., K. H. Park, and J. Seo, "Maximization of acoustic energy difference between two spaces", J. Acoust. Soc. Am. 128, 121-131 (2010).

[6] M. B. Møller, M. Olsen, and F. Jacobsen, "A hybrid method combining synthesis of a sound field and control of acoustic contrast", in Proceedings of the 132nd Audio Engineering Society Convention (2012).

[7] J.-H. Chang and F. Jacobsen, "Sound field control with a circular double-layer array of loudspeakers”, J. Acoust. Soc. Am. 131, 4518-4525 (2012).

[8] S. J. Elliott, J. Cheer, J.-W. Choi, and Y. Kim, "Robustness and regularization of personal audio systems.", IEEE Trans. Audio Speech Lang. Proc. 20, 2123-2133 (2012).

[9] O. Kirkeby, P. A. Nelson, F. Orduna-Bustamante, and H. Hamada, "Local sound field reproduction using digital signal processing”, J. Acoust. Soc. Am. 100, 1584-1593 (1996).

[10] P. Nelson, "A review of some inverse problems in acoustics", International Journal of Acoustics and Vibration 6, 118-34 (2001).

[11] J.-H. Chang, J.-Y. Park, and Y.-H. Kim, "Scattering effect on the sound focused personal audio system", J. Acoust. Soc. Am. 125, 3060-3066 (2009).

[12] J. Park, M. Song, J. Chang, and Y. Kim, "Performance degradation due to transfer function errors in acoustic brightness and contrast control: sensitivity analysis", in Proceedings of the 20th International Congress on Acoustics (2010).

[13] P. Jackson and F. Jacobsen, "Estimation of a region's acoustic planarity with a pressuremicrophone array", J. Acoust. Soc. Am. submitted (2012).

[14] M. A. Akeroyd, J. Chambers, D. Bullock, A. R. Palmer, A. Q. Summerfield, P. A. Nelson, and S. Gatehouse, "The binaural performance of a cross-talk cancellation system with matched or mismatched setup and playback acoustics", J. Acoust. Soc. Am. 121, 1056-1069 (2007).

[15] J. Francombe, R. Mason, M. Dewhirst, and S. Bech, "Determining the threshold of acceptability for an interfering audio programme", in Proceedings of the 132nd Audio Engineering Society Convention (2012). 